\title{
Regulation of muscle development by DPF3, a novel histone acetylation and methylation reader of the BAF chromatin remodeling complex
}

\author{
Martin Lange, ${ }^{1}$ Bogac Kaynak, ${ }^{1,8}$ Ulrike B. Forster, ${ }^{2}$ Martje Tönjes, ${ }^{1}$ Jenny J. Fischer, ${ }^{1}$ \\ Christina Grimm, ${ }^{1}$ Jenny Schlesinger, ${ }^{1}$ Steffen Just, ${ }^{3}$ Ilona Dunkel, ${ }^{1}$ Tammo Krueger, ${ }^{1}$ \\ Siegrun Mebus, ${ }^{4}$ Hans Lehrach, ${ }^{5}$ Rudi Lurz, ${ }^{6}$ Johan Gobom, ${ }^{7}$ Wolfgang Rottbauer, ${ }^{3}$ \\ Salim Abdelilah-Seyfried, ${ }^{2}$ and Silke Sperling ${ }^{1,9}$ \\ ${ }^{1}$ Group Cardiovascular Genetics, Department Vertebrate Genomics, Max Planck Institute for Molecular Genetics, Berlin \\ 14195, Germany; ${ }^{2}$ Cell Polarity and Epithelial Development, Max Delbrück Center, Berlin 13125 Germany; ${ }^{3}$ Molecular \\ Cardiology, Ruprecht-Karls-Universität Heidelberg, Heidelberg 69120, Germany; ${ }^{4}$ Department Pediatric Cardiology, \\ German Heart Center Berlin, Berlin 13353, Germany; ${ }^{5}$ Department Vertebrate Genomics, Max Planck Institute \\ for Molecular Genetics, Berlin 14195, Germany; ${ }^{6}$ Microscopy Unit, Max Planck Institute for Molecular Genetics, \\ Berlin 14195, Germany; ${ }^{7}$ Mass Spectrometry Group, Department Vertebrate Genomics, Max Planck Institute \\ for Molecular Genetics, Berlin 14195, Germany
}

Chromatin remodeling and histone modifications facilitate access of transcription factors to DNA by promoting the unwinding and destabilization of histone-DNA interactions. We present DPF3, a new epigenetic key factor for heart and muscle development characterized by a double PHD finger. DPF3 is associated with the BAF chromatin remodeling complex and binds methylated and acetylated lysine residues of histone 3 and 4. Thus, DPF3 may represent the first plant homeodomains that bind acetylated lysines, a feature previously only shown for the bromodomain. During development Dpf3 is expressed in the heart and somites of mouse, chicken, and zebrafish. Morpholino knockdown of $d p f 3$ in zebrafish leads to incomplete cardiac looping and severely reduced ventricular contractility, with disassembled muscular fibers caused by transcriptional deregulation of structural and regulatory proteins. Promoter analysis identified $D p f 3$ as a novel downstream target of Mef2a. Taken together, DPF3 adds a further layer of complexity to the BAF complex by representing a tissue-specific anchor between histone acetylations as well as methylations and chromatin remodeling. Furthermore, this shows that plant homeodomain proteins play a yet unexplored role in recruiting chromatin remodeling complexes to acetylated histones.

[Keywords: Heart and skeletal muscle development and function; PHD finger; BAF chromatin remodeling complex; SMARCD3-BAF60; acetylated and methylated histones; Mef2]

Supplemental material is available at http://www.genesdev.org.

Received January 16, 2008; revised version accepted July 1, 2008.

Complex transcription networks mediate cell specification, proliferation, and differentiation throughout development and life. Coordinated activation and repression of different subsets of genes is regulated at several levels by genetic and epigenetic mechanisms. Genomic DNA is packaged into nucleosomes, the basic unit of chromatin structure formed by DNA wrapped around a histone octamer. Chromatin remodeling and covalent histone

\footnotetext{
${ }^{8}$ Present address: Gladstone Institute of Cardiovascular Disease, San Francisco, CA 94158, USA.

${ }^{9}$ Corresponding author.

E-Mail sperling@molgen.mpg.de; FAX 40-30-8413-1699.

Article is online at http://www.genesdev.org/cgi/doi/10.1101/gad.471408.
}

modifications facilitate DNA access for DNA-binding transcription factors (Simone 2006; Bernstein et al. 2007; Sperling 2007). Specific patterns of histone tail modifications attract or repel regulatory proteins of the chromatin remodeling complex. Histone modifications can influence one another and thus not just the level of modification but also the pattern may dictate biological outcome (Fischer et al. 2008).

The main histone modifications are acetylation and methylation. Recently, several transcription or remodeling factors (e.g., TFIID, BPTF, Yng2) have been identified, which bind to methylated histone lysine residues via different domains, such as WD-40, Tudor, MBT, and the plant homeodomain (PHD) (Kim et al. 2006; Ruthenburg 
et al. 2007; Vermeulen et al. 2007). Acetylation of histone lysine residues by histone acetyltransferases (HATs) stimulates gene expression by recruiting chromatin remodeling complexes and neutralizing positive charge, resulting in destabilization of histone-histone and histone-DNA interactions that limit access of transcription factors to DNA. The effect of HATs is counteracted by histone deacetylases (HDACs), and represents a control point of gene expression exemplified by cardiac growth in response to acute and chronic stress stimuli (Backs and Olson 2006). The recruitment of remodeling complexes is highly affected by histone acetylation and the bromodomain is the only protein domain that is presently known to recognize acetylated lysine residues of histones (Mujtaba et al. 2007). Surprisingly, bromodomains can be dispensable in vivo, which suggests functional redundancy among proteins (Elfring et al. 1998; Bourachot et al. 1999; Hassan et al. 2002; Mohrmann and Verrijzer 2005). Chromatin remodeling complexes use free energy derived from ATP hydrolysis to actively alter nucleosomal structure. These factors peel DNA from the edge of the nucleosomes forming a DNA loop or slide the histone octamer to a different position (Kassabov et al. 2003). Different chromatin remodeling complexes have been identified (e.g., SWR/ NURF, CHD/NuRD, or SWI/SNF), which are defined by a unique subunit composition and the presence of a distinct ATPase (Palacios and Puri 2006; Simone 2006; Bao and Shen 2007). Mammalian SWI/SNF-like complexes (BAF complexes) are characterized by central core subunits BRG1 and BRM and 10 further subunit elements; e.g., SMARCD3 (BAF60c) representing a muscle-specific component. BRG1 and BRM contain an ATPase domain and a bromodomain that recognizes acetylated lysine in histone tails and other proteins (Sif 2004; Simone 2006). Thus, BRG1 acts as a ubiquitously expressed targeting molecule to anchor chromatin remodeling complexes on promoters with particular histone modification marks (Hassan et al. 2002, 2007). SMARCD3 is a promiscuous partner for several DNA-binding transcription factors, including nuclear receptors $\operatorname{PPAR} \gamma, \operatorname{RXR} \alpha, \operatorname{RAR}$, and muscle regulatory factors like MEF2, MyoD, Nkx2.5, Tbx5, and Gata4 (Debril et al. 2004; Lickert et al. 2004; Palacios and Puri 2006; Simone 2006; Flajollet et al. 2007; Z.Y. Li et al. 2007). Tissue-specific transcription can be initiated by ligand-dependent activation of signaling cascades; e.g., phosphorylation of SMARCD3 and MEF2 through p38 MAP-kinase leads to translocation of MEF2 to the nucleus, potentially enhances their interaction, and finally, the BAF complex is targeted to muscle-specific loci (Simone et al. 2004; Rauch and Loughna 2005).

In the early mouse embryo Smarcd3 is specifically expressed in heart and somites, and is required for cardiac looping and outflow tract development. Smarcd3-deficient mice furthermore show impaired trabeculation of the heart and disorganized somites (Lickert et al. 2004; Takeuchi et al. 2007). The four Mef2 transcription factors (Mef2a, Mef2b, Mef2c, and Mef2d) regulate muscle cell differentiation, and can, in part, compensate each other's function (Karamboulas et al. 2006). Mef2s are DNA-binding transcription factors that interact with members of the MyoD family to cooperatively activate muscle specific genes. Embryonic hearts of Mef2a-deficient mice and zebrafish show myofibrillar disarray, and mice with skeletal muscle ablation of Mef2c form abnormally assembled sarcomeres (Naya et al. 2002; Wang et al. 2005; Potthoff et al. 2007).

In a genome-wide gene expression study of congenital malformed human hearts we identified DPF3 as significantly up-regulated in the right ventricular myocardium of patients with Tetralogy of Fallot (TOF) (Kaynak et al. 2003). The study showed disease-associated expression profiles for a panel of cardiac conditions in addition to profiles specific for each cardiac chamber of the normal human heart. DPF3 contains a double PHD finger containing protein and a putative transcription factor. We show that DPF3 is associated with the BAF complex, and binds methylated and acetylated lysine residues of histone 3 and 4. Thus, DPF3 contains the first PHD that binds acetylated lysines, a feature previously only shown for bromodomains. Furthermore, Dpf3 shows tissue-specific expression in heart and somites during development of mouse, chicken, and zebrafish. Promoter analysis identified $D p f 3$ as a novel downstream target of Mef2a. Morpholino (MO) knockdown of $d p f 3$ in zebrafish lead to severely reduced cardiac contractility, incomplete cardiac looping and defective organization of cardiac and skeletal muscle fibers caused by transcriptional deregulation of structural and regulatory proteins essential for muscle fibers. Taken together, DPF3 adds a further layer of complexity to the BAF complex by representing a tissue-specific anchor between histone acetylations as well as methylations and chromatin remodeling.

\section{Results}

DPF3 is a muscle expressed member of the D4, zinc, and double PHD finger family

DPF3 is an evolutionary highly conserved member of the d4-protein family characterized by an $\mathrm{N}$-terminal 2/3 domain unique to this protein family, a C2H2-type zinc finger, and a C-terminal PHD zinc finger (Supplemental Table S1; Natalia et al. 2001). DPF3 gives rise to two splice variants (DPF3 $a$ and $D P F 3 b)$ in human and mouse, four in chicken, and one in zebrafish, with human and mouse DPF3 differing only by one amino acid (Supplemental Fig. S1). The human DPF3b variant and the DPF3 full-length ortholog in zebrafish had not been identified previously, and were cloned from human heart and zebrafish cDNA (AY803021, NM_001111169). DPF3 variants differ at the $\mathrm{C}$ terminus such that DPF3a encodes a 357-amino-acid protein containing a single truncated PHD finger, while DPF3b consists of 378 amino acids and a double PHD finger (Fig. 1A).

The other members of the $\mathrm{d} 4$ family are DPF1 and DPF2. In the mouse, Dpf1 (Neud4) is expressed predominantly in the brain, and may have an important role in developing neurons through regulation of cell survival as 
A

Figure 1. DPF3 - a zinc and double PHD finger protein. (A) Sequence conservation and divergence of human DPF3 isoforms. DPF3a (AAX20019.1) and DPF3b (NP_036206) contain an N-terminal 2/3 domain, a putative nuclear localization signal (NLS), a nuclear receptor interaction domain (NID), and a C2H2-Krüppel-like zinc finger. Note that the C-terminal double PHD is truncated in DPF3a. Cysteine and histidine residues of the PHDs are marked in bold. $(B, C)$ Expression of DPF3 mRNA in malformed and normal human hearts. Realtime PCR analysis of DPF3 mRNA levels in myocardial, right ventricular tissue from patients with TOF, ventricular septal defect (VSD), and healthy controls. Analysis of splice variant-specific expression of $D P F 3 a$ and $D P F 3 b$ in TOF patients and healthy controls. Expression values normalized to the housekeeping gene HPRT. Statistically significant differences analyzed by two-sided Wilcoxon test are indicated with asterisks $\left.\left(\left[{ }^{\star}\right] P<0.01 ;{ }^{\left.{ }^{*}\right]}\right] P<0.01\right)$. Scale bars represent \pm SEM. (D) Tissue-specific expression of DPF3 mRNA in humans analyzed by Northern blot. DPF3 mRNA expression is restricted to heart and skeletal muscle. The blot containing mRNA from the indicated tissues was probed with ${ }^{32} \mathrm{P}-D P F 3$ cDNA (top panel), stripped, and reprobed with ${ }^{32} \mathrm{P}$-Actin cDNA (bottom panel).

B

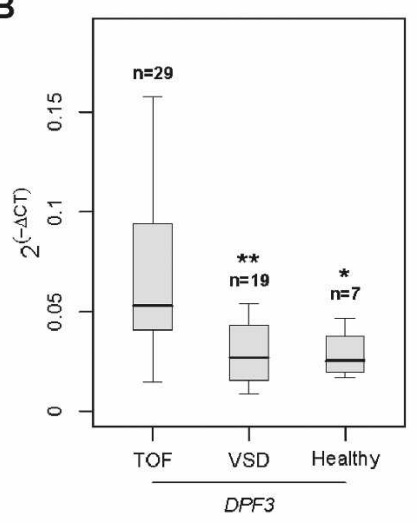

C

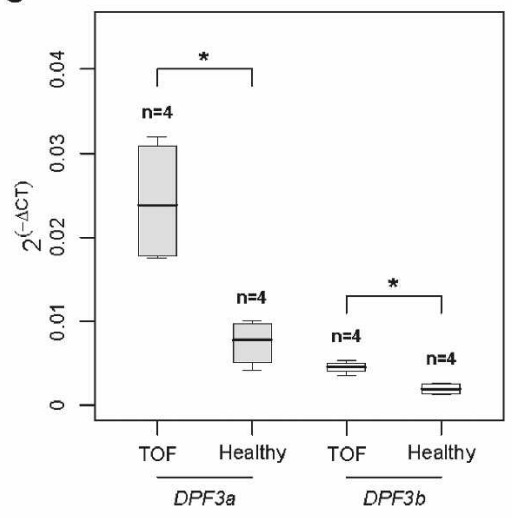

D

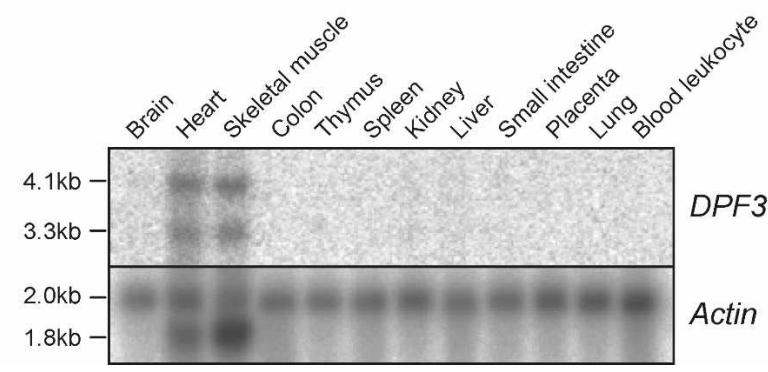

right ventricular systolic pressure. Using a multiple human tissue Northern blot we observed DPF3 to be specifically expressed in cardiac and skeletal muscle (Fig. 1D). death after deprivation of trophic factors (Gabig et al. 1994).

We found both splice variants of DPF3 to be significantly up-regulated in human right ventricular myocardial tissue of TOF hearts compared with age- and gendermatched samples obtained from hearts with single ventricular septal defects as well as healthy donors (Fig. $1 \mathrm{~B}, \mathrm{C})$. TOF represents a defect in heart looping and outflow tract formation characterized by a ventricular septal defect, an overriding aorta, right ventricular outflow tract stenosis and right ventricular hypertrophy secondary to hemodynamic stress, mainly due to increased

\section{DPF3a and DPF3b associate with BAF chromatin remodeling complexes}

DPF3 contains two PHD fingers, domains frequently found in nuclear proteins whose substrate tend to be nucleosomes (Bienz 2006). Using tandem affinity purification technique (TAP) and mass spectrometry we isolated potential nuclear binding partners of DPF3a and DPF3b in HEK293T cells. We identified nearly all core 
components of the BAF chromatin remodeling complex to be associated with both isoforms of DPF3 (Table 1). We found that a very high percentage of proteins purified with DPF3 correspond to the BAF complex $(91.2 \%$ BAF components with DPF3a and $86,8 \%$ with DPF3b as bait). Among the interactors of DPF3a and DPF3b we found SMARCD3, a heart and somite-specific subunit of the complex. To confirm the association of DPF3 with the BAF complex, we performed reverse-TAP and mass spectrometry using SMARCD3 as bait (Table 1). Thus, both DPF3 isoforms associate with the BAF chromatin remodeling complex.
DPF3 interacts with methylated and acetylated lysine residues of histones 3 and 4

It has recently become evident that proteins involved in chromatin remodeling recognize specific modifications on histone tails. The recognition of the methylation state of lysine residues on histone 3 and 4 has been shown to be mediated, among others, by the PHD, whereas lysine acetylations are recognized by the bromodomain (Kouzarides 2007). To address whether DPF3 generally binds to histones, we used a glutathione-Stransferase (GST) pull-down system and tested for the

Table 1. Human DPF3 protein interactions

\begin{tabular}{|c|c|c|c|c|c|c|c|c|c|}
\hline Bait & Alias & HUGO ID & $\begin{array}{l}\mathrm{MW} \\
(\mathrm{kDa})\end{array}$ & $\begin{array}{c}\text { Length } \\
\text { (amino acids) }\end{array}$ & $\begin{array}{l}\text { Mascot } \\
\text { score }\end{array}$ & $\begin{array}{l}\text { Spectral } \\
\text { counts }\end{array}$ & $\begin{array}{c}\text { Sequence } \\
\text { coverage }(\%)\end{array}$ & NSAF & $\begin{array}{c}\text { Accession } \\
\text { number }\end{array}$ \\
\hline \multirow[t]{14}{*}{ DPF3a } & BAF250A & ARID1A & 242.8 & 2285 & 1844 & 47 & 24 & 0.038 & O14497 \\
\hline & BAF250B & ARID1B & 237.1 & 2236 & 1171 & 33 & 14 & 0.027 & Q8NFD5 \\
\hline & BRG1 & SMARCA4 & 185.0 & 1647 & 2196 & 51 & 29 & 0.057 & P51532 \\
\hline & BRM & SMARCA2 & 181.3 & 1586 & 959 & 27 & 16 & 0.031 & P51531 \\
\hline & BAF170 & SMARCC2 & 133.2 & 1214 & 2160 & 48 & 30 & 0.073 & Q8TAQ2 \\
\hline & BAF155 & SMARCC1 & 123.2 & 1105 & 2401 & 49 & 38 & 0.081 & Q92922 \\
\hline & BAF60A & SMARCD1 & 55.2 & 476 & 80 & 4 & 11 & 0.014 & Q96GM5 \\
\hline & BAF60B & SMARCD2 & 52.7 & 456 & 846 & 23 & 44 & 0.093 & Q92925 \\
\hline & BAF60C & SMARCD3 & 55.2 & 483 & 644 & 19 & 36 & 0.072 & Q6STE5 \\
\hline & BAF57 & SMARCE1 & 46.7 & 411 & 982 & 19 & 49 & 0.085 & Q969G3 \\
\hline & BAF53 & ACTL6A & 47.9 & 429 & 826 & 17 & 39 & 0.073 & O96019 \\
\hline & BAF47 & SMARCB1 & 44.4 & 385 & 659 & 14 & 39 & 0.067 & Q12824 \\
\hline & $\beta$-actin & ACTB & 42.1 & 375 & 697 & 18 & 38 & 0.088 & P60709 \\
\hline & CERD4 & DPF3 & 26.1 & 224 & 844 & 15 & 63 & 0.123 & Q92784 \\
\hline \multirow[t]{14}{*}{ DPF3b } & BAF250A & ARID1A & 242.8 & 2285 & 1461 & 49 & 27 & 0.036 & O14497 \\
\hline & BAF250B & ARID1B & 237.1 & 2236 & 457 & 16 & 9 & 0.012 & Q8NFD5 \\
\hline & BRG1 & SMARCA4 & 185.0 & 1647 & 1912 & 43 & 22 & 0.044 & P51532 \\
\hline & BRM & SMARCA2 & 181.3 & 1586 & 792 & 21 & 12 & 0.022 & P51531 \\
\hline & BAF170 & SMARCC2 & 133.2 & 1214 & 1676 & 42 & 28 & 0.058 & Q8TAQ2 \\
\hline & BAF155 & SMARCC1 & 123.2 & 1105 & 1511 & 42 & 29 & 0.064 & Q92922 \\
\hline & BAF60A & SMARCD1 & 55.2 & 476 & 1062 & 26 & 45 & 0.091 & Q96GM5 \\
\hline & BAF60B & SMARCD2 & 52.7 & 456 & 957 & 21 & 43 & 0.077 & Q92925 \\
\hline & BAF60C & SMARCD3 & 55.2 & 483 & 616 & 19 & 37 & 0.066 & Q6STE5 \\
\hline & BAF57 & SMARCE1 & 46.7 & 411 & 969 & 19 & 44 & 0.077 & Q969G3 \\
\hline & BAF53 & ACTL6A & 47.9 & 429 & 699 & 15 & 35 & 0.059 & O96019 \\
\hline & BAF47 & SMARCB1 & 44.4 & 385 & 880 & 18 & 56 & 0.078 & Q12824 \\
\hline & $\beta$-actin & ACTB & 42.1 & 375 & 604 & 18 & 43 & 0.080 & P60709 \\
\hline & CERD4 & DPF3 & 26.1 & 224 & 909 & 16 & 63 & 0.120 & Q92784 \\
\hline \multirow[t]{14}{*}{ BAF60c } & BAF250A & ARID1A & 242.8 & 2285 & 680 & 46 & 23 & 0.046 & O14497 \\
\hline & BAF250B & ARID1B & 237.1 & 2236 & 375 & 30 & 12 & 0.031 & Q8NFD5 \\
\hline & BAF180 & PBRM1 & 194.1 & 1689 & 91 & 9 & 5 & 0.012 & Q86U86 \\
\hline & BRG1 & SMARCA4 & 185.0 & 1647 & 523 & 39 & 21 & 0.054 & P51532 \\
\hline & BRM & SMARCA2 & 181.3 & 1586 & 195 & 20 & 10 & 0.029 & P51531 \\
\hline & BAF170 & SMARCC2 & 133.2 & 1214 & 656 & 35 & 25 & 0.066 & Q8TAQ2 \\
\hline & BAF155 & SMARCC1 & 123.2 & 1105 & 1069 & 42 & 36 & 0.087 & Q92922 \\
\hline & BAF60B & SMARCD2 & 52.7 & 456 & 91 & 5 & 9 & 0.025 & Q92925 \\
\hline & BAF60C & SMARCD3 & 55.2 & 483 & 202 & 14 & 19 & 0.066 & Q6STE5 \\
\hline & BAF57 & SMARCE1 & 46.7 & 411 & 711 & 23 & 38 & 0.127 & Q969G3 \\
\hline & BAF53 & ACTL6A & 47.9 & 429 & 258 & 15 & 36 & 0.080 & O96019 \\
\hline & BAF47 & SMARCB1 & 44.4 & 385 & 195 & 14 & 39 & 0.083 & Q12824 \\
\hline & $\beta$-actin & АСТВ & 42.1 & 375 & 398 & 13 & 37 & 0.079 & P60709 \\
\hline & CERD4 & DPF3 & 26.1 & 224 & 72 & 2 & 11 & 0.021 & Q92784 \\
\hline
\end{tabular}

Peptides associated with DPF3a, DPF3b, and BAF60c identified by TAP and mass spectrometry. (MW) Calculated molecular weight. (NSAF) Normalized spectral abundance factor (Florens et al. 2006). 
ability of recombinant full-length GST-DPF3 to pull down histones from calf thymus extracts followed by Western analyses using histone specific antibodies against $\mathrm{H} 2 \mathrm{~A}, \mathrm{H} 2 \mathrm{~B}, \mathrm{H} 3$, and H4. DPF3b was able to pull down histones $\mathrm{H} 3$ and $\mathrm{H} 4$ but not histones $\mathrm{H} 2 \mathrm{~A}$ and $\mathrm{H} 2 \mathrm{~B}$, whereas DPF3a did not bind any histones (Fig. 2A). To further analyze if DPF3b binds specific histone modifications through its PHD fingers, we tested a broad panel of histone 3 and 4 peptides harboring specific modifications such as methylations, acetylations, or phosphorylations on different residues with pull-down assays (Fig. 2B). Surprisingly, we observed specific binding of DPF3b to acetylated lysines on histone 3 and 4 (H3K14ac, H3K9ac, H4K5ac, H4K8ac, H4K12ac, H4K16ac) besides binding to mono- and dimethylated Lys 4 on histone $3(\mathrm{H} 3 \mathrm{~K} 4 \mathrm{mel} / 2)$. Unmodified histone 3 and 4 and other modifications were detected at the background level.

Since DPF3b contains a double PHD finger, we asked whether the PHD1 or PHD2 alone is sufficient to recognize histone lysine modifications. Pull-down assays revealed that single DPF3-PHD fingers were sufficient for the interaction with lysine acetylations on histone 4, whereas histone 3 acetylations and methylations were only recognized by the double PHD finger (Fig. 2B). Furthermore, DPF3a, which only contains a truncated PHD finger, did not bind any of the studied peptides. To substantiate these findings, we generated point mutations of residues essential for the structural integrity of the aromatic cage formed by the PHD finger (W358E) as well as residues that contribute to zinc-complexing (C360R/ C363R). These mutations lead to the abolishment of single and double PHD finger binding to $\mathrm{H} 3$ and $\mathrm{H} 4$ modified peptides showing the specificity of the interactions (Fig. 3B). The binding properties of DPF3-PHD fingers were furthermore compared with the known methyl- and acetyllysine recognition of the BPTF-PHD finger and the BRG1 bromodomain.

\section{Mapping of DPF3-binding sites reveals global colocalization with histone modifications by chromatin immunoprecipitation (ChIP)-chip}

To obtain a global overview of potential downstream targets of DPF3, we used ChIP followed by array detection
A

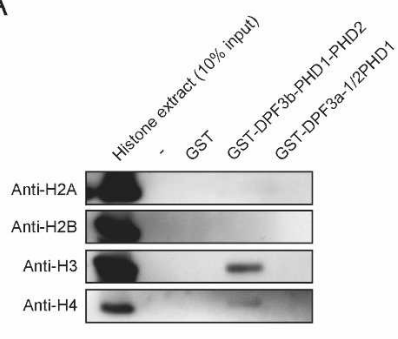

C

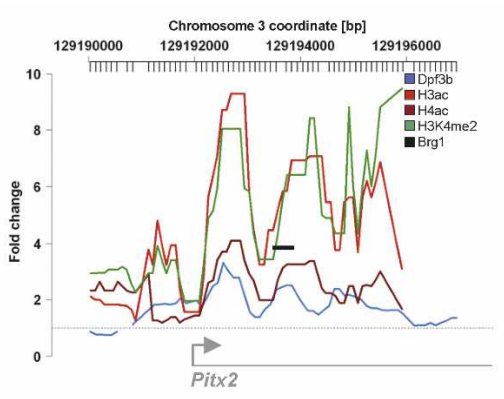

B

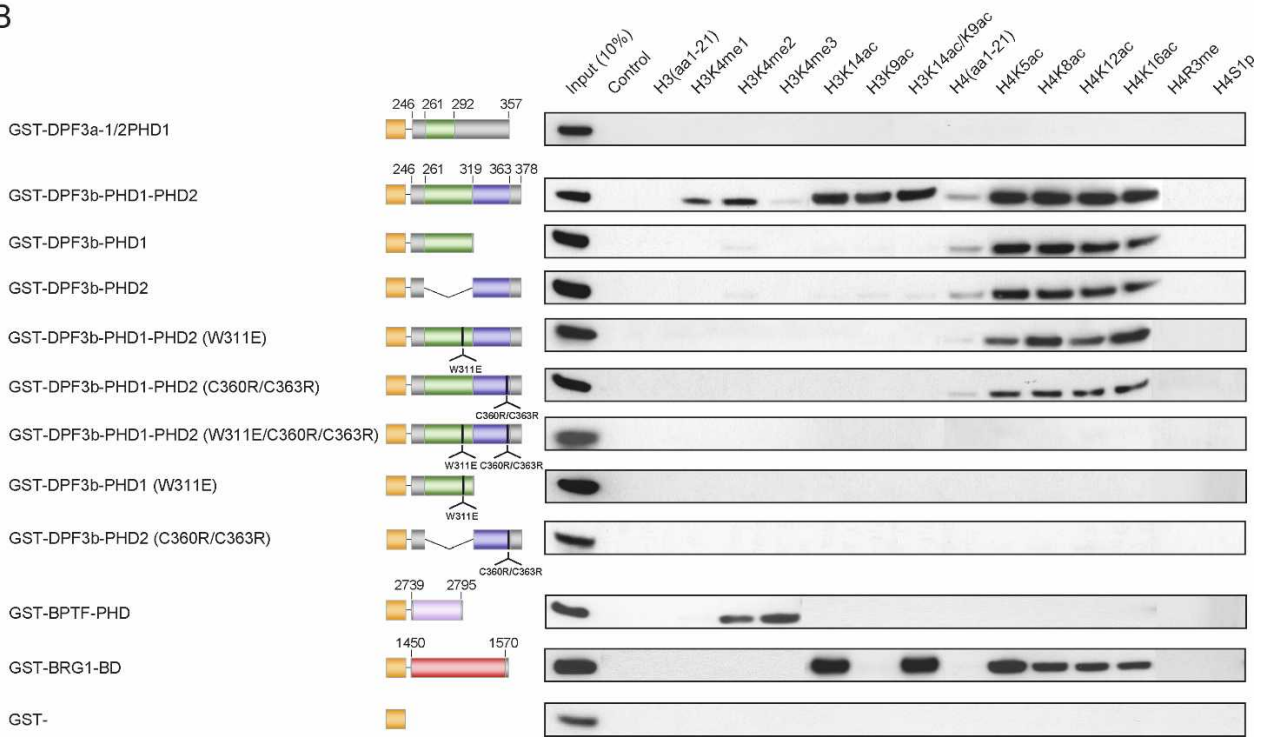

Figure 2. The PHD fingers of DPF3b interact with modified histone tails on histone 3 and histone 4. $(A)$ Pull-down assays followed by Western blotting and immunodetection of indicated histones using GST-DPF3 fusion proteins and calf thymus histone extracts. (B) Western blot analysis of histone peptide pulldowns with indicated GST-DPF3 fusion proteins and biotinylated peptides. GST-BPTF and GST-BRG1 fusion proteins are shown as positive/negative controls. (Orange) GST tag; (green) DPF3-PHD1; (blue) DPF3-PHD2; (purple) BPTF-PHD; (red) BRG1 bromodomain. $(C)$ Co-occurrence of Dpf3, BRG1, H3K4me2, and H3ac/H4ac modifications on the murine Pitx2 locus. Normalized and smoothed relative ChIP-chip intensities and position of real-time PCR primer are shown. 


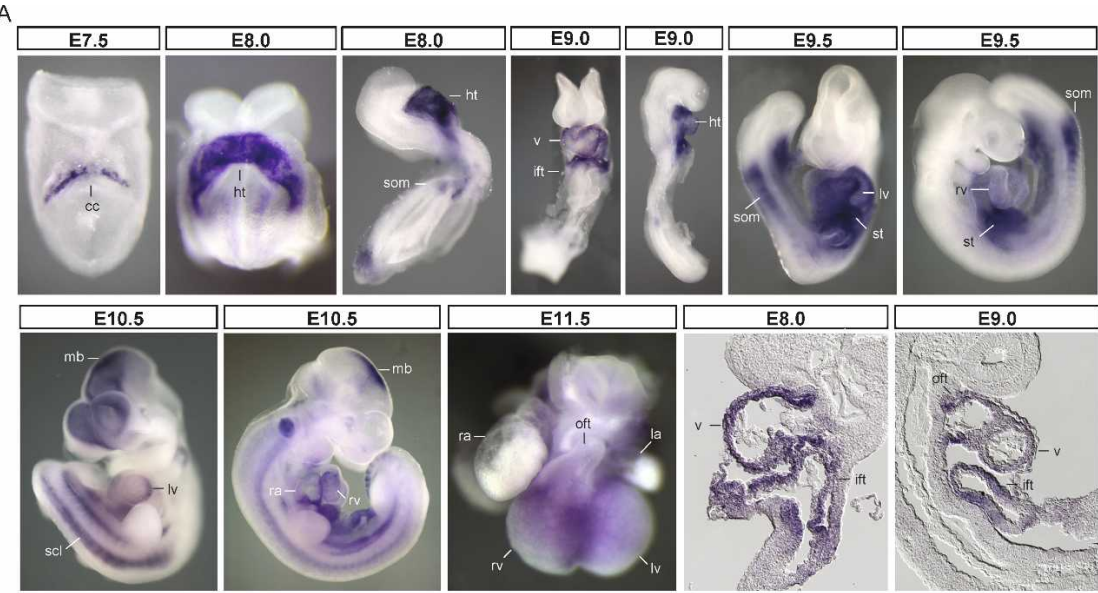

B

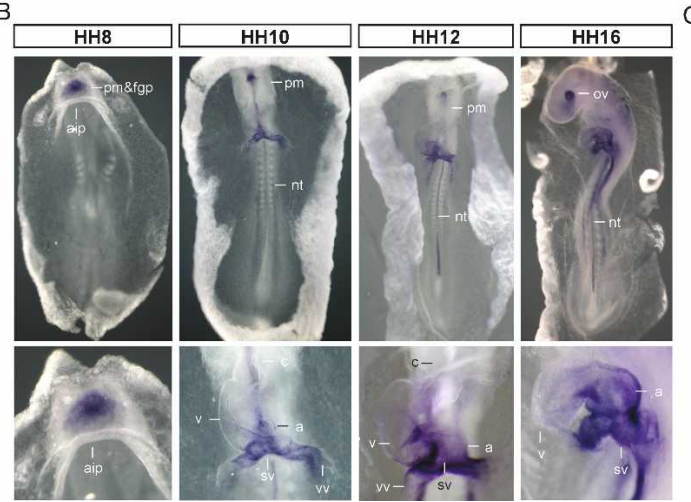

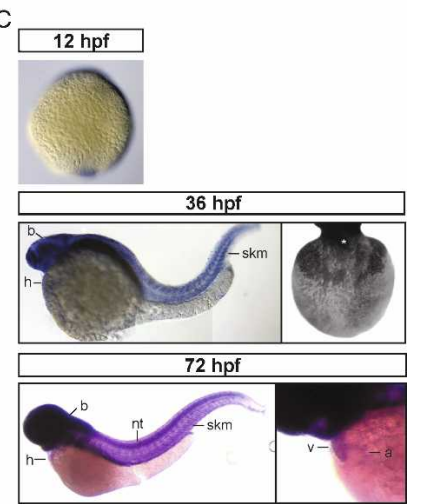

Figure 3. Expression patterns of $D p f 3$ mRNA during embryonic development analyzed by in situ hybridization. Expression pattern of $D p f 3$ mRNA during mouse $(A)$, chicken $(B)$, and zebrafish $(C)$ development is shown (ventral and lateral views and closeups). (cc) Cardiac crescent; (ht) heart tube; $(\mathrm{v})$ ventricle; (ift) inflow tract; (oft) outflow tract; (lv) left ventricle; (rv) right ventricle; (la) left atrium; (ra) right atrium; (som) somites; (st) septum transversum; (mb) midbrain; (scl) sclerotome; (pm) prechordal mesoderm; (fgp) foregut pocket; (aip) anterior intestinal portal; (sv) sinus venosus; (vv) vitelline veins; (a) atrium; (nt) neural tube; (c) conus; (h) heart; (v) ventricle; (skm) skeletal muscle; (ov) optic vesicle.
(ChIP-chip) and mapped the genomic localization of DPF3-binding sites in C2C12 skeletal muscle cells. We designed a custom muscle specific promoter array with 740,000 probes covering $10 \mathrm{~kb}$ upstream of and $3 \mathrm{~kb}$ downstream from $\sim 12,000$ transcripts. This array enabled analysis of our genes of interest with a much higher degree of tiling and sequence coverage than standard whole-genome arrays would provide. We found a total of 1201 transcripts in close distance to DPF3a- or DPF3b-binding sites $(460$ and 979 respectively; 238 shared) (Supplemental Table S2). To gain first insight into the functional role of downstream targets of DPF3, we performed an analysis of GO terms and found that DPF3 targets particularly play a role in cell proliferation, nucleosome assembly, and chromatin remodeling (Supplemental Table S3). Moreover, DPF3b targets are especially important for cardiovascular development and cytoskeleton organization. A number of DPF3 targets are structural genes, like $\alpha$ actinin (Actn1), cardiomyopathy associated 3 (Cmya3), myosin light chain (Myl1), and troponin $C$ (Tnnc1); muscle-regulating transcription factors and cofactors such as myocyte enhancer factor (Mef2c/d), Cbp/p300-interacting transactivator (Cited2), paired-like homeodomain transcription factor 2 (Pitx2), four and $a$ half LIM domains 2 (Fhl2), inhibitor of DNA binding 2 (Id2); as well as genes essential for muscle differentiation like bone morphogenetic protein 2 (Bmp2).

Figure 2C exemplifies observed binding sites of DPF3b in the vicinity of target genes such as Pitx2. Moreover,
Pitx2 represents an example of co-occurrence of DPF3bbinding sites with acetylated/methylated lysine residues of histone 3 and 4, which have been analyzed by us previously (Fischer et al. 2008). To gain insight into the frequency and relevance of DPF3b binding to histone 3 and 4 modification marks, we compared the two ChIPchip data sets. Out of 546 DPF3b-binding sites, 265 overlapped with histone 3 acetylation, 220 overlapped with histone 4 acetylation, and 294 overlapped with histone 3 methylation marks. Thus, $66 \%$ of DPF3b-binding sites overlap with acetylation marks and $54 \%$ with methylation marks, which is significantly more than one could expect from random permutations (mininum 26\%, maximum $39 \%$ ).

\section{Co-occurrence of modified histones with DPF3} and BRG1 genomic binding sites

Considering that DPF3 is a member of the BAF chromatin remodeling complex and binds modified histones, we additionally analyzed the co-occurrence of DPF3-binding sites with those of BRG1, a core component of the BAF complex. To select potentially shared targets, we performed ChIP-chip analysis for BRG1 in C2C12 cells (data not shown). Using real-time PCR we screened 21 muscle relevant downstream targets for co-occurring binding sites of modified histones, DPF3 and BRG1, and observed a high degree of overlap (Table 2). This suggests 
Table 2. Co-occurrence of DPF3b with histone modifications and BRG1 binding

\begin{tabular}{|c|c|c|c|c|c|c|c|c|c|}
\hline \multicolumn{2}{|l|}{ Gene } & \multicolumn{3}{|c|}{ Peak position } & \multicolumn{2}{|l|}{ Brg1 } & \multicolumn{2}{|l|}{$\mathrm{Dpf} 3 \mathrm{~b}$} & \multirow[b]{2}{*}{ Histone modifications } \\
\hline Name & Ensemble transcript ID & Chr. & Start & End & Fold change & SD & Fold change & SD & \\
\hline Jmjd1c & ENSMUST00000095573 & 10 & 66581196 & 66581787 & 18.00 & 0.12 & 7.12 & 0.10 & $\mathrm{H} 3 \mathrm{ac}$ \\
\hline Ctnnbl & ENSMUST00000007130 & 9 & 120783627 & 120783820 & 5.84 & 0.09 & 3.79 & 0.08 & Н3ac, Н3К4me2 \\
\hline Musk & ENSMUST00000098059 & 4 & 58380142 & 58380250 & 5.25 & 0.17 & 3.27 & 0.06 & H3ac, H3K4me2 \\
\hline Flrt2 & ENSMUST00000057324 & 12 & 96093975 & 96094272 & 3.81 & 0.13 & 2.75 & 0.04 & $\mathrm{H} 3 \mathrm{ac}, \mathrm{H} 3 \mathrm{~K} 4 \mathrm{me} 2$ \\
\hline Gsk3b & ENSMUST00000023507 & 16 & 38010138 & 38010438 & 6.07 & 0.14 & 2.54 & 0.04 & Н3ac, Н3К4me2 \\
\hline Cald1 & ENSMUST00000079391 & 6 & 34529598 & 34530598 & 11.44 & 0.19 & 2.36 & 0.12 & H3ac, H3K4me2 \\
\hline Pten & ENSMUST00000013807 & 19 & 32825230 & 32825724 & 2.16 & 0.14 & 2.16 & 0.30 & H3ac, H3K4me2 \\
\hline Creb1 & ENSMUST00000049932 & 1 & 64468558 & 64468747 & 2.09 & 0.12 & 1.84 & 0.05 & Н3ac, Н3К4me2 \\
\hline Arpc2 & ENSMUST00000006467 & 1 & 74172324 & 74172916 & 3.72 & 0.18 & 1.50 & 0.10 & Н3ac, Н3К4me2 \\
\hline Sema3a & ENSMUST00000095012 & 5 & 13405946 & 13406745 & 4.50 & 0.87 & 5.67 & 0.15 & $\mathrm{H} 3 \mathrm{ac}, \mathrm{H} 4 \mathrm{ac}$ \\
\hline Zeb2 & ENSMUST00000028229 & 2 & 44933122 & 44933520 & 6.23 & 0.30 & 6.71 & 0.20 & H3ac, H4ac, H3K4me2 \\
\hline Trim 23 & ENSMUST00000022225 & 13 & 105298442 & 105298742 & 49.82 & 0.14 & 4.82 & 0.15 & H3ac, H4ac, H3K4me2 \\
\hline Pitx2 & ENSMUST00000029657 & 3 & 129193542 & 129193945 & 3.97 & 0.05 & 4.77 & 0.08 & $\mathrm{H} 3 \mathrm{ac}, \mathrm{H} 4 \mathrm{ac}, \mathrm{H} 3 \mathrm{~K} 4 \mathrm{me} 2$ \\
\hline Asb5 & ENSMUST00000033918 & 8 & 56048828 & 56049632 & 13.41 & 0.48 & 4.05 & 0.11 & H3ac, H4ac, H3K4me2 \\
\hline Foxp1 & ENSMUST00000074346 & 6 & 99060857 & 99061758 & 10.99 & 0.02 & 2.94 & 0.11 & H3ac, H4ac, H3K4me2 \\
\hline Csrp2 & ENSMUST00000020403 & 10 & 110335474 & 110335974 & 8.03 & 0.17 & 2.06 & 0.09 & $\mathrm{H} 3 \mathrm{ac}, \mathrm{H} 4 \mathrm{ac}, \mathrm{H} 3 \mathrm{~K} 4 \mathrm{me} 2$ \\
\hline Cxcr7 & ENSMUST00000065587 & 1 & 92036250 & 92036550 & 22.29 & 0.29 & 1.93 & 0.23 & H3ac, H4ac, H3K4me2 \\
\hline Daam 1 & ENSMUST00000085299 & 12 & 72801340 & 72801838 & 10.00 & 0.09 & 2.73 & 0.12 & H3K4me2 \\
\hline Igfbp5 & ENSMUST00000027377 & 1 & 72811484 & 72812274 & 4.59 & 0.05 & 2.31 & 0.04 & $\mathrm{H} 4 \mathrm{ac}$ \\
\hline Lamc1 & ENSMUST00000027752 & 1 & 155062722 & 155063616 & 1.51 & 0.18 & 2.01 & 0.09 & $\mathrm{H} 4 \mathrm{ac}$ \\
\hline Mtss1 & ENSMUST00000080371 & 15 & 58894578 & 58895274 & 2.43 & 0.17 & 1.64 & 0.10 & $\mathrm{H} 4 \mathrm{ac}$ \\
\hline
\end{tabular}

Real-time PCR analysis showing cobinding of DPF3b and BRG1 at genomic sites that are further characterized by histone modifications. (SD) Standard deviation.

that DPF3 potentially serves as an anchor between the BAF complex and modified histones.

\section{Dpf3 expression patterns during embryonic development}

As DPF3 was up-regulated in hypertrophic cardiac tissue of TOF patients, we were interested in its spatiotemporal expression pattern during embryogenesis and performed in situ hybridization in mouse, chicken, and zebrafish embryos. Whole-mount in situ hybridization in mouse embryos revealed cardiac and somite expression of Dpf3a starting in the first differentiating cardiomyocytes of the cardiac crescent at embryonic day 7.5 (E7.5) and in the first somites at E8.0 (Fig. 3A). A detailed description is provided in the Supplemental Material. Section in situ hybridization revealed that $D p f 3 a$ expression was restricted to the myocardial compartment of the heart (Fig. 3A). Further in situ hybridizations using a common Dpf3 probe revealed a similar expression pattern (data not shown).

In order to analyze expression profiles of $D p f 3 a$ and $D p f 3 b$ during later stages of heart development, realtime PCR analysis was performed using cDNA obtained from embryonic hearts extracted between E9.5 and E16.5 as well as P0 and adult hearts. Expression of Dpf3a and $D p f 3 b$ was detectable from E9.5 onward, although $D p f 3 a$ showed substantially higher expression untilE 11.5 , where both splice variants subsequently reached a similar level of expression that remained stable until birth and adulthood (Supplemental Fig. S2).

The expression patterns of Dpf1 and Dpf2 were also analyzed by in situ hybridization in mouse embryos. $D p f 1$ was predominantly expressed in the developing brain, whereas Dpf2 was ubiquitously expressed (data not shown).

In situ hybridization experiments in chicken embryos using a probe targeting all splice variants of $D p f 3$ showed conservation of the mouse $D p f 3$ expression pattern (Fig. 3B; see the Supplemental Material for a detailed description). In zebrafish embryos, $d p f 3$ was strongly expressed within the developing brain and throughout somitic tissues along the entire length of the embryonic trunk and tail shown by in situ hybridization at 36 and $72 \mathrm{~h}$ postfertilization (hpf) (Fig. 3C). Within the heart, $d p f 3$ was strongly expressed in the ventricle and faintly in the atria. In the early embryo at $12 \mathrm{hpf}$, dpf3 is expressed unspecifically. (Fig. 3C). Expression of $d p f 2$ at $36 \mathrm{hpf}$ is within the developing brain and spinal cord (data not shown), and in contrast to $d p f 3$ was not detected in heart or somites. This suggests that $d p f 3$ is likely the only muscle expressed $\mathrm{d} 4$ family member. Taken together, these data demonstrate an evolutionarily conserved expression pattern of DPF3 orthologs.

Knockdown of dpf3 reveals its essential role for heart and skeletal muscle development in vivo

To address the role of $d p f 3$ in vivo, we performed MO antisense oligonucleotide-mediated knockdown in zebrafish. We characterized embryos injected with $\mathrm{MO}^{d p f 3}$, which targets the exon4-intron4 boundary of $d p f 3$ premRNA and blocks correct splicing. The specificity of the $\mathrm{MO}^{d p f 3}$ was demonstrated by coinjection of synthetic 
and mature $d p f 3$ mRNA, resulting in rescue of the $\mathrm{MO}^{d p f 3}$ phenotypes (Fig. 4). Efficacy of the $\mathrm{MO}^{d p f 3}$ was tested by PCR, which showed that the majority of $d p f 3$
mRNA was incorrectly spliced leading to two truncated proteins (Fig. 4A; Supplemental Material).

To assess cardiac morphogenesis and differentiation,
A

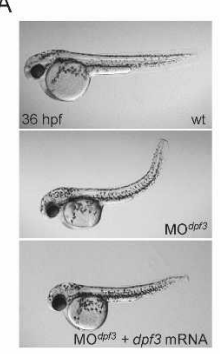

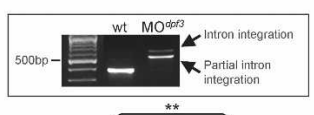

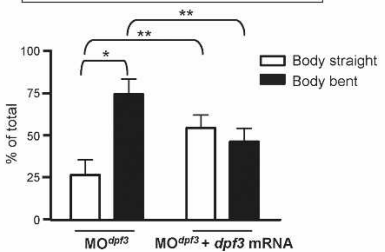

C
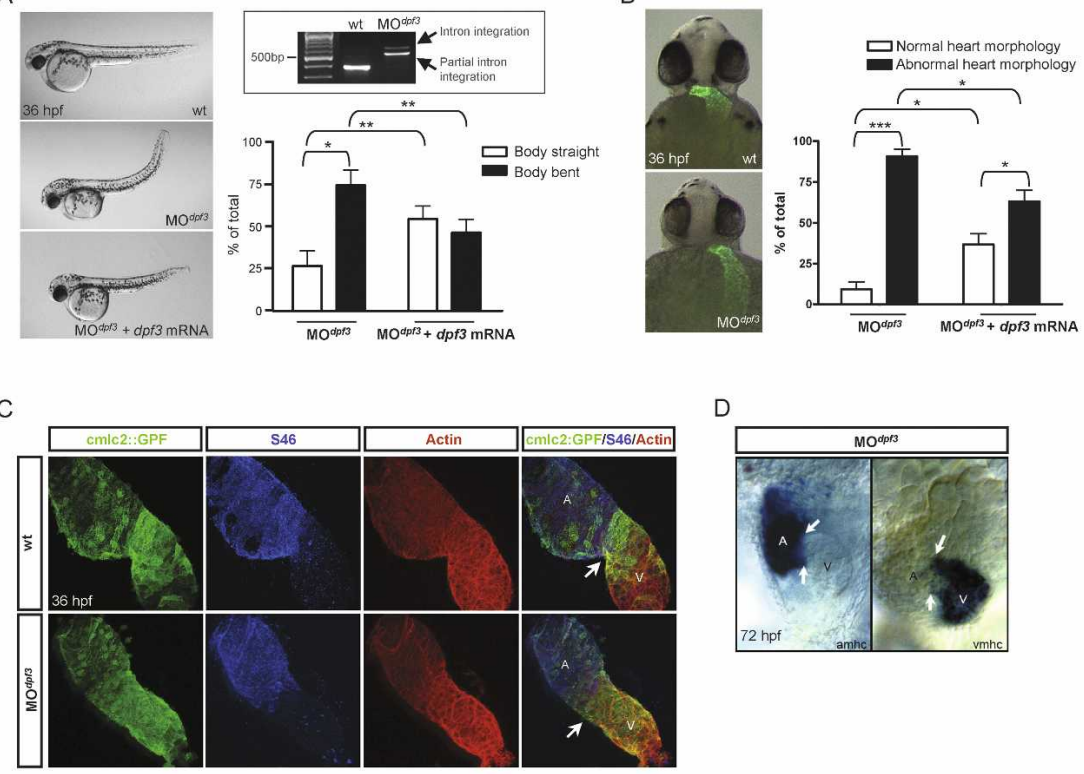

D
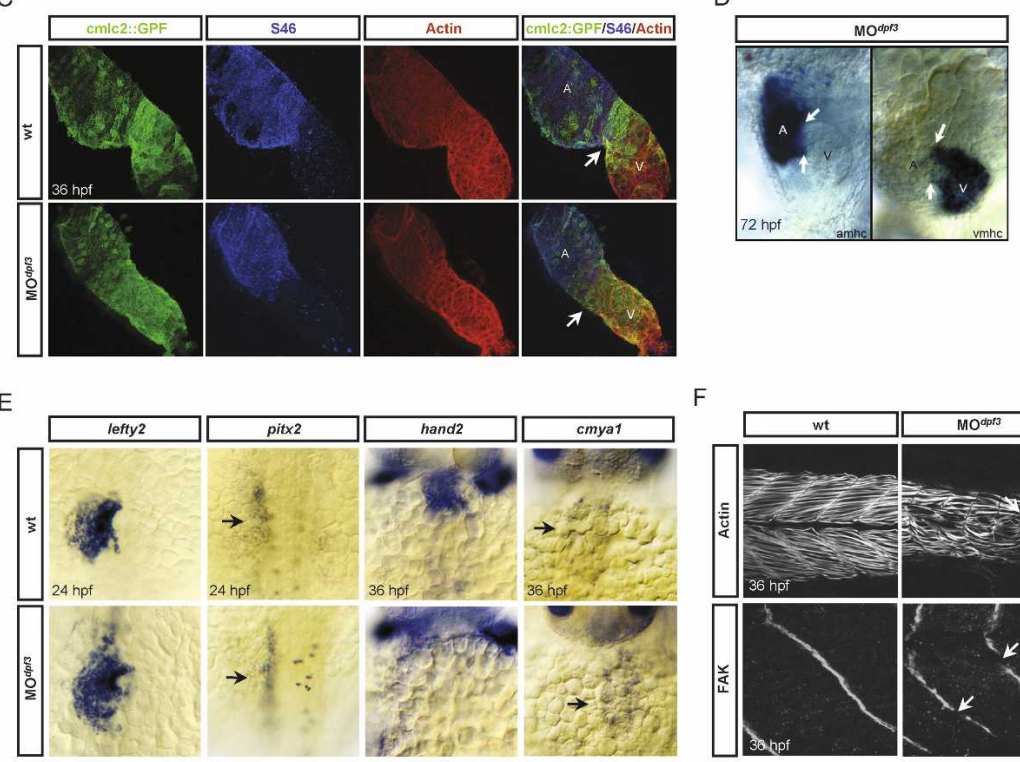

$\mathrm{F}$

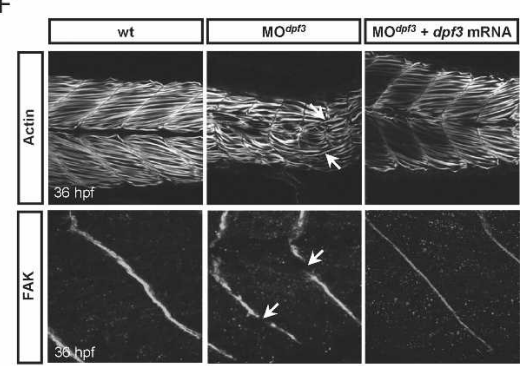

G

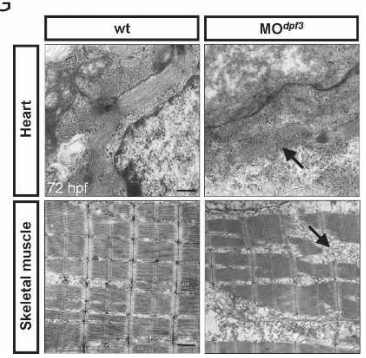

$\mathrm{H}$

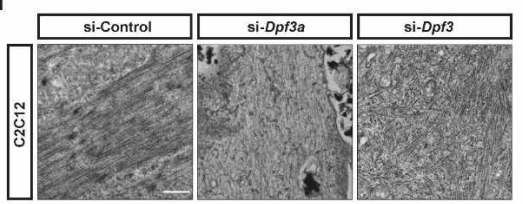

Figure 4. Knockdown of $d p f 3$ in zebrafish and in $\mathrm{C} 2 \mathrm{C} 12$ mouse skeletal muscle cells analysis of body and heart morphology in embryos injected with $\mathrm{MO}^{d p f 3}$ and controls at $36 \mathrm{hpf}$. $(A)$ Knockdown of $d p f 3$ lead to abnormal body posture (curved tail) in zebrafish embryos. The phenotype could be rescued by coinjection of mature $d p f 3-m R N A$. (Five independent experiments; embryos scored: 255 MO, $321 \mathrm{MO}+$ rescue RNA; $\left.\left.\left[^{\star}\right] P<0.05 ;{ }^{\star \star}{ }^{\star}\right] P<0.01.\right)(B)$ Analysis of heart morphology in $[T g(c m l c:: G F P)]$ zebrafish embryos. Knockdown of $d p f 3$ lead to abnormal heart looping, which could be rescued by coinjection of mature $d p f 3$-mRNA. (Three independent experiments; embryos scored: $101 \mathrm{MO}, 145 \mathrm{MO}+$ rescue RNA; $\left.\left.\left.{ }^{\star}\right] P<0.05 ;{ }^{\star \star \star}\right] P<0.001.\right)$ Statistical significance analyzed by two-way ANOVA with Bonferroni post hoc testing. Scale bars represent \pm SEM. $(C)$ Analysis of isolated zebrafish hearts by confocal microscopy. Cmlc marks all cardiomyocytes, S46 labels, the cells of the atrium, actin is predominantly expressed in the ventricle at $36 \mathrm{hpf}$. (D) In situ hybridization of chamber-specific markers amhc and vmhc. (E) In situ hybridization of left-right asymmetry marker lefty2, and pitx2 as well as of differentially expressed genes hand2 and cmya1. (F) Analysis of skeletal muscle in wild-type, dpf3 morphant, and rescued embryos by immunohistochemistry at $36 \mathrm{hpf}$. (Top panel) Actin staining shows myofibrillar disarray and transversion of somite boundaries. (Bottom panel) FAK staining reveals disruption of somite boundaries in $d p f 3$ morphants. $(G)$ Disrupted sarcomere integrity in heart and skeletal muscle of $d p f 3$ morphant embryos at 72 hpf shown by electron microscopy. Bar, $500 \mathrm{~nm} .(H)$ siRNA-mediated knockdown of $D p f 3 a$ and $D p f 3$ lead to defects in myofibrillar assembly in C2C12 mouse skeletal muscle cells analyzed by electron microscopy. Bar, $500 \mathrm{~nm}$. 
we used the $\mathrm{MO}^{d p f 3}$ in a transgenic line of zebrafish that expresses green fluorescent protein (GFP) under control of the cardiac myosin light chain 2 (cmlc2) promoter region $[\mathrm{Tg}(\mathrm{cmlc2:GFP})]$. Injection of $\mathrm{MO}^{d p f 3}$ at the onecell stage resulted in $91 \%$ of embryos with abnormal heart morphology $(n=101)$ and in $74 \%$ of embryos with a curved tail at $36 \mathrm{hpf}(n=255)$ (Fig. 4A,B). Consistent with strong somitic expression of $d p f 3, \mathrm{MO}^{d p f 3}$-injected embryos frequently displayed disturbed forward swimming movements indicating skeletal muscle defects (Fig. $4 \mathrm{~A})$. Coinjection of synthetic full-length $d p f 3$ mRNA produced a significant rescue effect, with the percentage of embryos with a $d p f 3$ morphant body phenotype decreasing to $46 \%(n=321, P<0.01)$ and the heart phenotype decreasing to $63 \%(n=145, P<0.05)$ (Fig. $4 A, B)$. The heart phenotype was characterized by a thin and elongated heart tube, with both ventricular and atrial portions being affected. Moreover, looping of the heart was strongly reduced and the atrioventricular boundary was poorly defined in morphants (Fig. 4A,C). The strength of ventricular and atrial contractility was weakened compared with wild type, which resulted in slower blood flow, supported also by a significantly reduced ventricular shortening fraction (VSF) $(P<0.05)$ (data not shown). Nevertheless, the heart beat rate was normal (Supplemental Movies S1, S2). Both myocardial and endocardial layers were formed in morphant embryos, excluding defects in endocard-myocard signaling (data not shown).

In order to characterize the cardiac phenotype more thoroughly, we analyzed isolated hearts using confocal microscopy and found that despite the weakly developing atrioventricular boundary and loss of heart looping, atrial and ventricular myocyte specification was grossly normal (Fig. 4C). Immunohistochemistry using the atrial specific marker S46 showed that the atrium was clearly separated from the ventricle (Yelon et al. 1999). This finding was further confirmed by normal atrial myosin heavy chain (amhc) and ventricular myosin heavy chain (vmhc) expression at $72 \mathrm{hpf}$ analyzed by in situ hybridization (Fig. 4D).

\section{Dpf3 morphant zebrafish embryos display muscle fiber disarray}

To identify genes deregulated in $d p f 3$ morphants, we performed gene expression analysis (Affymetrix GeneChip Zebrafish Genome Arrays) using RNA from whole $d p f 3$ morphant embryos with severely reduced ventricular contractility and control-injected stage-matched embryos ( $n=30$, two replicates). Genes differentially regulated with an adjusted $P$-value of $<0.1$ were selected (1210 of 15,000 transcripts) for global functional analysis based on overrepresented Gene Ontology terms (Supplemental Table S4). The set of up-regulated genes contained many genes essential for transcriptional regulation, nucleosome assembly, and metabolic processes, whereas genes involved in ion and electron transport were overrepresented among down-regulated genes. A subset of differentially expressed genes was confirmed by real-time PCR including genes directly involved in sarcomere assembly and muscle function that could explain the cardiac and skeletal muscle phenotypes of $d p f 3$ morphants (Supplemental Table S5). We observed significantly increased expression of cmya1 (fold change 2.9) and of actin-binding protein 280-like (flncb; fold change 2.5). Furthermore, we found decreased expression of heart and neural crest derivatives expressed 2 (hand2; fold change 0.5), thymosin $\beta$ (fold change 0.3 ), and a novel protein (zgc:101755) similar to mouse actin filament capping protein of muscle Z-lines (fold change $0.5)$. However, as gene expression profiling was performed using whole embryos, we further analyzed expression levels in situ. Figure 4E shows in situ hybridization analysis confirming the differential expression of hand2 and cmya1. To test if the heart looping defects were due to disturbed establishment of left-right asymmetry in the embryo, asymmetrically expressed markers left-right determination factor 2 (lefty2) and pitx2 were analyzed revealing that left-right asymmetry was properly initiated.

To further evaluate the deregulation of sarcomeric proteins we performed immunohistochemistry of morphant muscle fibers in the zebrafish and found a grossly disturbed actin organization compared with wild-type animals. The normal chevron-shaped somite organization was lost and myofibers were misaligned. Frequently, myofibers transversed somite boundaries. Focal adhesion kinase $(\mathrm{FAK})$ is a marker of somite boundaries. Immunohistochemistry using an antibody against FAK showed disruption of somite boundaries (Fig. 4F). The thickness of somites was also markedly reduced. The specificity of this phenotype was confirmed by coinjection of synthetic full-length $d p f 3$ mRNA together with $\mathrm{MO}^{d p f 3}$, which largely restored the myofiber organization and somite boundary formation (Fig. 4F).

Using transmission electron microscopy, we found that few myofibrils were present in $d p f 3$ morphant ventricles and skeletal muscle, which displayed a severe disruption of sarcomere assembly. Analysis of $D p f 3$ siRNA knockdown in $\mathrm{C} 2 \mathrm{C} 12$ mouse skeletal muscle cells showed conservation of this phenotype (Fig. 4G,H) with myofibrillar disarray compared with fiber aggregation in cells treated with control siRNA.

\section{Mef2a regulates Dpf3 expression in vivo}

Mef2a-deficient mice and zebrafish embryos are phenotypically similar to the observed myofibrillar disarray in dpf3 knockdown embryos (Naya et al. 2002; Wang et al. 2005; Potthoff et al. 2007). Consequently, we screened the Dpf3 proximal promoter for potential Mef2-binding sites. Within a conserved $1.2 \mathrm{kbp}$ promoter region we found three Mef2 matrices using TRANSFAC MATCH with stringent settings (Fig. 5A; Kel et al. 2003). Mef2a ChIP-chip analysis in mouse cardiomyocytes (HL-1 cells) showed a significant peak of Mef2a binding in the $D p f 3$ promoter region that could also be confirmed by real-time PCR (1.8-fold change) (Fig. 5A). Knockdown of Mef2a in HL-1 cells using two different siRNAs led to a 
A
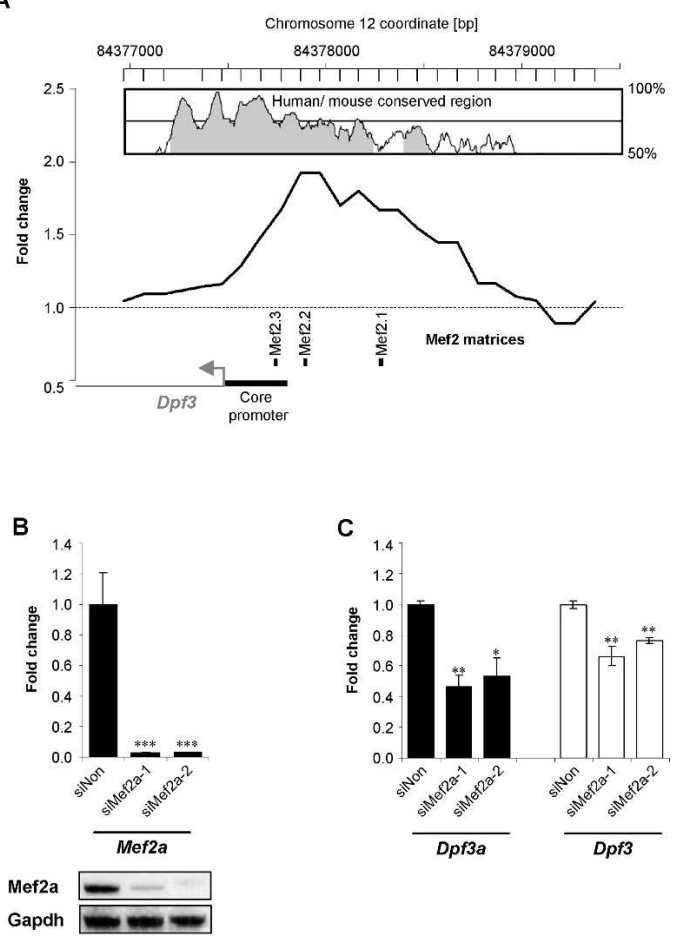

D

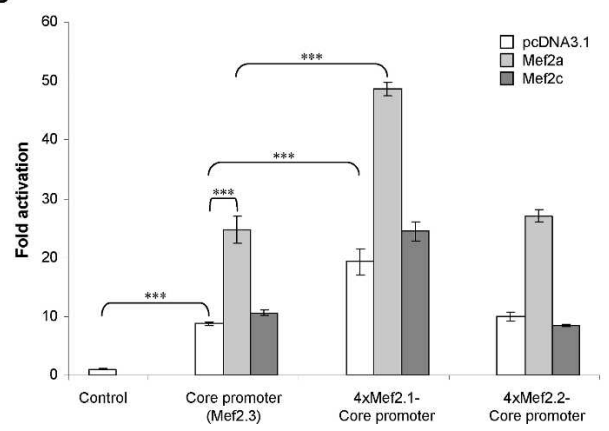

Figure 5. Mef2a regulates DPF3 expression. (A) ChIP followed by chip analysis shows binding of Mef2a to an evolutionary conserved region of the $D p f 3$ promoter in vivo. Mef2a matrices obtained by TRANSFAC MATCH are indicated. Conservation of promoter sequence is shown. The $D p f 3$ core promoter indicated is a minimal sufficient region required for transcriptional activity. (B) Knockdown of Mef2a in HL-1 cells using two different siRNAs. Knockdown efficiency was analyzed by realtime PCR and Western blot. (siNon) Nonspecific/scrambled. $(C)$ Knockdown of Mef2a in HL-1 cells lead to reduced expression of $D p f 3$ and $D p f 3 a$. (siNon) Nonspecific/scrambled; $\left(^{\star}\right) P<0.05 ;\left(^{\star \star}\right) P<0.01 ;\left(^{\star \star \star}\right) P<0.001$. $(D)$ Luciferase reportergene assay using the DPF3 core promoter alone or in combination with four repeats of the conserved, putative Mef2-binding sites (Mef2.1, Mef2.2, Mef2.3) fused to luciferase. Activity of the reporter was measured alone or in cotransfections with Mef2a/Mef2c expression vectors in HEK293T cells.

reduction of $D p f 3$ expression of up to $40 \%$, demonstrating that Mef2a functionally binds the $D p f 3$ promoter and activates its expression (Fig. 5B,C). Transcriptional regulation of $D p f 3$ by Mef2a was also tested in luciferase reportergene assays using promoter fusion constructs of a previously characterized DPF3 core promoter (M. Lange and S. Sperling, unpubl.) and four consecutive repeats of the putative Mef2-binding sites. Cotransfections in HEK293T cells revealed an activation of the core promoter by Mef2a, which was additionally enhanced by the Mef2.1-binding site, supporting a role for Mef2a as a regulator of $D p f 3$ through combinatorial effects on the Mef2.1 and Mef2.3 sites (Fig. 5D).

\section{Discussion}

Targeting of the BAF chromatin remodeling complex to specific chromatin sites

A central question regarding the action of chromatin remodeling complexes is how they are recruited to their target nucleosomes at specific positions within the genome. Most likely two mechanisms, the guidance by DNA-binding transcription factors and the binding to acetylated histone tails, play a central role (Peterson and Workman 2000; Hassan et al. 2001). Both transcription factor-binding sites as well as acetylated histones do not occur exclusively in conjunction with actively transcribed genes; thus, potentially, the interplay and co-occurrence of both might be essential for directed and tissue-specific gene transcription. Here, we present DPF3, which contains the first PHD fingers shown to bind acetylated in addition to methylated histone residues. Moreover, DPF3 links these modifications to the BAF chromatin remodeling complex and displays an essential role for skeletal and cardiac muscle development and function in vivo. The tissue-specific expression of DPF3 in combination with the specific read-out of modified histone residues allows for a side-directed recruitment of the BAF chromatin remodeling complex, similar to that of DNA-binding transcription factors.

The high impact of the modification status of histones (acetylation/deacetylation) on transcription and on the phenotype is well characterized; e.g., class II HDACs control cardiac growth and gene expression in response to stress stimuli (Backs and Olson 2006). DPF3 potentially represents the missing link to explain the high impact of the histone modification status on the recruitment of the BAF complex to chromatin target sites. So far, only bromodomains, frequently found in core and subunit proteins of chromatin remodeling complexes, have been shown to recognize histone acetylation marks.

Using ChIP we show on a global scale that DPF3 binds distinct chromatin sites in vivo, which are furthermore essential for muscle development and function, and marked by acetylated and/or methylated histones. It would be interesting to analyze if DPF3 is also associated with histone-modifying enzymes through the BAF complex or other interaction partners. Thus, the binding of DPF3 would be followed by a change in the histone modification status, building a regulatory feedback loop. 
The PHD of DPF3 binds modified histone lysine residues

PHDs are frequently found in nuclear proteins, and are defined by a stretch of $\sim 60$ amino acids containing conserved cysteine and histidine residues (C4-H-C3) that coordinate two zinc ions forming interweaved zinc fingers bridged by two small $\beta$-strands (Bienz 2006). They are known to serve as a protein-protein interaction domain and bind nuclear phosphoinositides as well as nucleosomes (Bienz 2006; Ruthenburg et al. 2007). Moreover, in a proteome-wide screen, only eight out of 18 PHD fingers showed specific histone methyl-lysine interactions, indicating additional roles for the PHD (Shi et al. 2007). We report that the double PHD finger of DPF3 interact with acetylated as well as methylated histone tail residues, namely acetylated lysines on histones 3 and 4 (H3K9ac, H3K14ac, H4K5ac, H4K8ac, H4K12ac, $\mathrm{H} 4 \mathrm{~K} 16 \mathrm{ac})$ and mono- and dimethylated lysine on histone 3 (H3K4me1/me2). Interestingly, single PHD fingers of DPF3 only recognize histone 4 acetylation and an intact PHD finger is necessary for histone interactions, as the truncated PHD1 of DPF3a is not capable of binding any histones.

So far, single PHD fingers have been shown to recognize methylated histones; e.g., the PHD fingers of BPTF and ING2 (Shi et al. 2006; Wysocka et al. 2006) bind H3K4me with increasing affinity according to methylation status, while BHC80-PHD recognizes unmodified H3K4 (Lan et al. 2007). Moreover, methylation at different residues, namely $\mathrm{H} 3$ methylated at both Lys 4 and Arg 2, can be read simultaneously by a single PHD of RAG2, revealing additional complexity in the readout of combinatorial modifications (Ramon-Maiques et al. 2007). Further experiments are needed to answer the question if binding of acetylation and methylation marks by the double PHD finger of DPF3 can occur simultaneously, which would allow a combinatorial readout of different modifications.

The finding that $\mathrm{H} 3$ modifications are only recognized by the double PHD finger may be due to the interweaved nature of the PHD finger. The domain necessary for $\mathrm{H} 3 \mathrm{~K} 4 \mathrm{me} 1 / \mathrm{me} 2$ and $\mathrm{H} 3 \mathrm{ac}$ recognition might be a compound in which amino acids from PHD1 and PHD2 contribute to the three-dimensional structure.

Histone methyl-lysine-binding properties similar to DPF3 have been described for the malignant brain tumor (MBT) domain of L3MBTL1 and a mutated form of BPTFPHD, which also specifically recognize H3K4me1/me2. Although structurally unrelated, both domains achieve methyl-lysine binding through formation of a cage consisting of aromatic residues (H. Li et al. 2007; Min et al. 2007). The PHD fingers of DPF3 contain several aromatic residues that can potentially contribute to the formation of an aromatic cage, although a conserved tryptophan is missing.

Further experiments using crystallography and NMR spectroscopy will determine the structural basis for the histone tail recognition by DPF3.
Role of DPF3 in heart and skeletal muscle development

The up-regulated expression of DPF3 in patients with TOF, a congenital heart defect in part characterized by muscular hypertrophy, prompted us to investigate its role during development and muscle differentiation. Knockdown in zebrafish embryos and RNAi in mouse skeletal muscle cells revealed an essential role of Dpf3 in muscle cell differentiation.

In morphant embryos, we frequently observed myofibrillar disarray, transversion of the somite boundary by actin filaments, and disruption of somite boundary formation. In particular, the $z$-disc of sarcomeres representing the lateral boundaries where titin, nebulin, and the thin filaments are anchored (Clark et al. 2002), appeared to be affected. This phenotype could be explained by the deregulation of several genes essential for muscle fiber function shown by our expression studies-e.g., capZ $\alpha-1$ (zgc:101755) and tropomodulin 4 (Schafer et al. 1995; Sussman et al. 1998/_the actin-binding protein filamin $c \gamma b$ (flncb) and its interaction partner cmya1. Filamin $C$-knockout mice display severe defects in myogenesis, including loss of distinct z-discs (Dalkilic et al. 2006), while Cmya1- $\alpha$-null mouse hearts show intercalated disc disruption and myofilament disarray (GustafsonWagner et al. 2007). Further, $d p f 3$ morphants frequently displayed impaired cardiac contractility, which may be due to the strong up-regulation of troponin I. Notably, mice expressing mutated versions of Troponin I display hypercontractility (James et al. 2000), mirror imaging the dpf3 morphant phenotype.

The morphant phenotype was also characterized by disturbed heart looping and a poorly defined AV boundary. Initial microarray analyses point to the deregulation of transcription factors and extracelluar matrix molecules implicated in heart looping and left-right asymmetry (data not shown). These molecules will be subject to further studies on the role of $d p f 3$ in early heart development. Notably, knockdown of Smarcd3, the DPF3 interaction subunit of the BAF complex, also affects heart looping in mouse and zebrafish by influencing Notch signaling (Takeuchi et al. 2007). Moreover, Bmp2, a gene essential for development of the AV cushions (Ma et al. 2005) is a target of Dpf3 in C2C12 cells analyzed by ChIP, and has been shown to be upstream of mef2 $a$ in zebrafish in a pathway controlling cardiac contractility (Wang et al. 2007).

Interestingly, the $d p f 3$ morphant phenotype resembles in part the defects seen in mef2a morphants and Mef2adeficient mice (Naya et al. 2002; Wang et al. 2005). As our experiments show that Mef2a regulates $D p f 3$, it is suggestive that the Mef2a phenotypes are partially caused by loss of Dpf3 function. In the future, it will be interesting to test the influence of Dpf3 on the Mef2a phenotypes in mouse and zebrafish in detail.

Despite the strong expression of $d p f 3$ in neuronal cells, we did not observe any obvious malformations of the brain. It has been shown recently that Dpf3a and Dpf1 seem to have overlapping functions during differentia- 
tion of neurons (Lessard et al. 2007). It is likely that Dpf1 may compensate for the loss of Dpf3 there, while expression in striated muscle appears exclusive to Dpf3.

We report that DPF3 contains the first PHDs known to bind acetylated as well as methylated histone residues, interacts with the BAF complex, and has an essential role for muscle development and function. It is tempting to speculate that DPF3a and DPF3b might serve as tissue-specific BAF subunits that regulate the transition of muscle precursors to differentiating myocytes. Moreover, it is highly suggestive that other PHD fingers might be capable to bind acetylation marks and play a yet unappreciated role in recruiting chromatin remodeling complexes.

\section{Materials and methods}

Detailed procedures are provided in the Supplemental Material

\section{Samples and preparation}

Human cardiac samples were obtained from the German Heart Center and treated as described (Kaynak et al. 2003). Mouse embryonic and adult hearts were dissected from the rest of the body at indicated stages and handled as human samples.

\section{Gene expression analyses}

Real-time PCR analysis was performed using SYBR Green I PCR Master Mix (Abgene) and the ABI PRISM 7900HT Sequence Detection System. Primer sequences are given in Supplemental Table S6. In situ hybridization in mouse, chicken, and zebrafish embryos was carried out as described (Wilkinson and Nieto 1993; Jowett and Lettice 1994). A multiple tissue human Northern blot (NTM 12, Clontech) was hybridized with a ${ }^{32}$ P-labeled cDNA probe against DPF3 (AY803021; 7-423 bp) according to the manufacturers' instructions.

Affymetrix GeneChip Zebrafish Genome Arrays were hybridized with labeled cDNA obtained from total RNA of MO-dpf3 and MO-control-injected zebrafish embryos 72 hpf. Four chips were hybridized (two MO-control, two MO- ${ }^{d p \nmid 3}$, 30 embryos each) (www.ebi.ac.uk/arrayexpress, E-TABM-354). Data were normalized via qspline after MAS background correction using the Bioconductor affy package and the zebrafish annotation package. Differentially expressed genes were calculated via the limma package. $P$-values were adjusted for multiple testing using the Benjamini and Hochberg method. Genes with an adjusted $P$-value of $<0.1$ were defined as differentially expressed.

\section{Antisense oligonucleotide $\mathrm{MO}$ and rescue experiments}

Full-length zebrafish $d p f 3$ (NM_001111169) was cloned into the $\mathrm{pCS}^{+}$expression vector and used as rescue construct. Constructs were transcribed using the SP6 MessageMachine kit (Ambion). For functional and rescue experiments, wild-type Tuebingen LF/AB hybrids; $\mathrm{Tg}(\mathrm{cmlc2}$ :GFP) transgenic fish embryos were injected with $\sim 75$ pg of mRNA. MOs (GeneTools) were injected at a concentration of $100 \mu \mathrm{mol} / \mathrm{L}$.

\section{Confocal and live-stream imaging}

Confocal images and z-stacks were obtained using the Zeiss Meta 510 confocal microscope with a $40 \times$ lens and $1 \times$ zoom. For live-stream imaging, $\mathrm{Tg}(\mathrm{cmlc2:GFP})$ transgenic embryos were prepared as described (Westerfield 1994). Myocardial contrac- tion and beating of the developing heart tube was imaged with a CoolSnap ES camera (Photometrics) on an Axioplan2 microscope.

Immunohistochemistry and transmission electron microscopy

Antibody staining was performed as described previously (Huang et al. 2003). Zebrafish electron micrographs were obtained essentially as described (Rottbauer et al. 2001). C2C12 cells were grown on Thermanox coverslips (13 mm ø; Nunc) and embedded in Spurr's resin. Sixty-nanometer sections were observed using Philips CM100 at $100 \mathrm{kV}$ (FEI Company) with a TVIPS Fastscan CCD camera (Tietz Systems).

\section{Proteomic analyses}

GST-DPF3 fusion proteins were created using the pGEX3 $\mathrm{x}$ vector, expressed in Escherichia coli BL21 DE3 pRARE and purified using glutathione-sepharose matrix (Amersham) according to the manufacturer's instructions.

For histone peptide-binding assays, $1 \mu \mathrm{g}$ of biotinylated histone peptide (Upstate Biotechnologies, and kind gifts of D. Patel and D. Allis) was incubated with $1 \mu \mathrm{g}$ of purified GST fusion protein in binding buffer (50 mM Tris- $\mathrm{HCl} 7.5,300 \mathrm{mM} \mathrm{NaCl}$, $0.1 \% \mathrm{NP}-40,50 \mu \mathrm{M} \mathrm{ZnAc)}$ overnight at $4^{\circ} \mathrm{C}$ with rotation. Streptavidin beads (Dynabeads) were added and incubated for 1 $\mathrm{h}$ at $4^{\circ} \mathrm{C}$ with rotation followed by four rounds of $15 \mathrm{~min}$ washing in binding buffer. Bound proteins were analyzed on SDSPAGE gels and subjected to immunoblotting analysis.

TAP was performed essentially as described (Gingras et al. 2005). Full-length DPF3a, DPF3b, and SMARCD3 was cloned into the pcDNA3-NTAP vector, verified by sequencing, and transfected into HEK293T cells.

\section{siRNA knockdown experiments}

C2C12 or HL-1 cells were seeded in six-well plates and transfected with $4.4 \mu \mathrm{L}$ of $20 \mu \mathrm{m}$ siRNA (Supplemental Table S7). siRNAs targeting $D p f 3 a$ (Invitrogen), both splice variants of Dpf3 (Qiagen), or a control siRNA (AllStars Negative Control siRNA, Qiagen) were used in $\mathrm{C} 2 \mathrm{C} 12$, and siRNAs targeting Mef2a in HL-1 cells. XtremeGene (Roche) and Lipofectamine Plus (Invitrogen) were used for transfection according to manufacturer's protocol and cultivated for $48 \mathrm{~h}$. Cells were subsequently subjected to electron microscopy or microarray gene expression analysis.

\section{ChIP with chip detection (ChIP-chip)}

C2C12 myoblasts cells were used either untransfected or transfected with Flag-empty, Flag-DPF3a or Flag-DPF3b expression vectors using Lipofectamine Plus (Invitrogen) according to manufacturers' instructions (Supplemental Fig. S3). ChIP experiments were performed in duplicate essentially as described (Horak et al. 2002). For immunoprecipitation, mouse-M2-antiFlag (Sigma) antibody and Brg1 (Santa Cruz Biotechnologies, sc-10768) antibodies were used at 10 and $5 \mu \mathrm{g} / \mathrm{mL}$ for $\mathrm{C} 2 \mathrm{C} 12$ cells and rabbit anti-Mef2A (Santa Cruz Biotechnologies) at 2 $\mu \mathrm{g} / \mathrm{mL}$ for HL-1 cells. Samples were labeled and hybridized according to NimbleGen standard procedures on custom designed muscle arrays (www.ebi.ac.uk/arrayexpress, A-MEXP-893). Array analysis was performed as described (Toedling et al. 2007). Enriched targets (23 sites) of the negative control (Flag-empty) were subtracted from DPF3 ChIP data. Data are deposited at www.ebi.ac.uk/arrayexpress (E-TABM362). 


\section{Reporter gene assays}

Reporter constructs were made by cloning four repeats of the putative Mef2-binding site upstream of a 385-bp (chr14:72,430,563-72,430,943) DPF3 minimal promoter into the pGL3 basic vector (Promega). Transient cotransfections were carried out in triplicates in 96-well plates in HEK293T cells by transfecting $45 \mathrm{ng}$ of reporter vector, $5 \mathrm{ng}$ of Firefly luciferase vector for internal normalization of transfection efficiency, and $100 \mathrm{ng}$ of the respective expression vectors. Activity was measured by Dual-Luciferase Assay (Promega) after $48 \mathrm{~h}$.

\section{Acknowledgments}

We gratefully acknowledge Drs. Bruneau, Gingras, Black, and Patel for providing plasmids; Drs. Allis and Patel for modified histone peptides; Dr. Yelon for amhc and vmhc probes; Dr. Schmidt for C2C12 cell line; Dr. Claycomb for the HL-1 cell line; and Dr. Huber and J. Toedling for bioinformatic help. We are indebted to B. Lukaszewska-McGreal and G. Lueder for technical assistance, M. Schueler for bioinformatic support, and Dr. Nordhoff for mass spectrometry support. We are very grateful to all patients and family members with congenital heart disease supporting our work. This work was supported by a grant to S.S. from the European Community's Sixth Framework Programme contract ("HeartRepair") LSHM-CT-2005-018630. Furthermore, M.T. receives a Ph.D. scholarship of the Studienstiftung des Deutschen Volkes. The work of J.G. was funded by the Structural Fonds of the European Union (project 2D/3DProteinChips) and the BMBF/NGFN project SMP Protein.

\section{References}

Backs, J. and Olson, E.N. 2006. Control of cardiac growth by histone acetylation/deacetylation. Circ. Res. 98: 15-24.

Bao, Y. and Shen, X. 2007. SnapShot: Chromatin remodeling complexes. Cell 129: 632.e1-632.e2. doi: 10.1016/j.cell.2007. 04.018.

Bernstein, B.E., Meissner, A., and Lander, E.S. 2007. The mammalian epigenome. Cell 128: 669-681.

Bienz, M. 2006. The PHD finger, a nuclear protein-interaction domain. Trends Biochem. Sci. 31: 35-40.

Bourachot, B., Yaniv, M., and Muchardt, C. 1999. The activity of mammalian brm/SNF2 $\alpha$ is dependent on a high-mobilitygroup protein I/Y-like DNA binding domain. Mol. Cell. Biol. 19: 3931-3939.

Clark, K.A., McElhinny, A.S., Beckerle, M.C., and Gregorio, C.C. 2002. Striated muscle cytoarchitecture: An intricate web of form and function. Annu. Rev. Cell Dev. Biol. 18: 637-706.

Dalkilic, I., Schienda, J., Thompson, T.G., and Kunkel, L.M. 2006. Loss of FilaminC (FLNc) results in severe defects in myogenesis and myotube structure. Mol. Cell. Biol. 26: 6522-6534.

Debril, M.B., Gelman, L., Fayard, E., Annicotte, J.S., Rocchi, S., and Auwerx, J. 2004. Transcription factors and nuclear receptors interact with the SWI/SNF complex through the BAF60c subunit. J. Biol. Chem. 279: 16677-16686.

Elfring, L.K., Daniel, C., Papoulas, O., Deuring, R., Sarte, M., Moseley, S., Beek, S.J., Waldrip, W.R., Daubresse, G., DePace, A., et al. 1998. Genetic analysis of brahma: The Drosophila homolog of the yeast chromatin remodeling factor SWI2/SNF2. Genetics 148: 251-265.

Fischer, J.J., Toedling, J., Krueger, T., Schueler, M., Huber, W., and Sperling, S. 2008. Combinatorial effects of four histone modifications in transcription and differentiation. Genomics 91: 41-51.

Flajollet, S., Lefebvre, B., Cudejko, C., Staels, B., and Lefebvre, P. 2007. The core component of the mammalian SWI/SNF complex SMARCD3/BAF60c is a coactivator for the nuclear retinoic acid receptor. Mol. Cell. Endocrinol. 270: 23-32.

Florens, L., Carozza, M.J., Swanson, S.K., Fournier, M., Coleman, M.K., Workman, J.L., and Washburn, M.P. 2006. Analyzing chromatin remodeling complexes using shotgun proteomics and normalized spectral abundance factors. Methods 40: 303-311.

Gabig, T.G., Mantel, P.L., Rosli, R., and Crean, C.D. 1994. Requiem: A novel zinc finger gene essential for apoptosis in myeloid cells. J. Biol. Chem. 269: 29515-29519.

Gingras, A.-C., Aebersold, R., and Raught, B. 2005. Advances in protein complex analysis using mass spectrometry. J. Physiol. 563: 11-21.

Gustafson-Wagner, E.A., Sinn, H.W., Chen, Y.L., Wang, D.Z., Reiter, R.S., Lin, J.L., Yang, B., Williamson, R.A., Chen, J., Lin, C.I., et al. 2007. Loss of $\mathrm{mXin} \alpha$, an intercalated disk protein, results in cardiac hypertrophy and cardiomyopathy with conduction defects. Am. J. Physiol. Heart Circ. Physiol. 293: $\mathrm{H} 2680-\mathrm{H} 2692$.

Hassan, A.H., Neely, K.E., and Workman, J.L. 2001. Histone acetyltransferase complexes stabilize swi/snf binding to promoter nucleosomes. Cell 104: 817-827.

Hassan, A.H., Prochasson, P., Neely, K.E., Galasinski, S.C. Chandy, M., Carrozza, M.J., and Workman, J.L. 2002. Function and selectivity of bromodomains in anchoring chromatin-modifying complexes to promoter nucleosomes. Cell 111: 369-379.

Hassan, A.H., Awad, S., Al-Natour, Z., Othman, S., Mustafa, F., and Rizvi, T.A. 2007. Selective recognition of acetylated histones by bromodomains in transcriptional co-activators. Biochem. J. 402: 125-133.

Horak, C.E., Mahajan, M.C., Luscombe, N.M., Gerstein, M., Weissman, S.M., and Snyder, M. 2002. GATA-1 binding sites mapped in the $\beta$-globin locus by using mammalian chIpchip analysis. Proc. Natl. Acad. Sci. 99: 2924-2929.

Huang, C.J., Tu, C.T., Hsiao, C.D., Hsieh, F.J., and Tsai, H.J. 2003. Germ-line transmission of a myocardium-specific GFP transgene reveals critical regulatory elements in the cardiac myosin light chain 2 promoter of zebrafish. Dev. Dyn. 228: 30-40.

James, J., Zhang, Y., Osinska, H., Sanbe, A., Klevitsky, R., Hewett, T.E., and Robbins, J. 2000. Transgenic modeling of a cardiac troponin I mutation linked to familial hypertrophic cardiomyopathy. Circ. Res. 87: 805-811.

Jowett, T. and Lettice, L. 1994. Whole-mount in situ hybridizations on zebrafish embryos using a mixture of digoxigeninand fluorescein-labelled probes. Trends Genet. 10: 73-74.

Karamboulas, C., Dakubo, G.D., Liu, J., De Repentigny, Y., Yutzey, K., Wallace, V.A., Kothary, R., and Skerjanc, I.S 2006. Disruption of MEF2 activity in cardiomyoblasts inhibits cardiomyogenesis. J. Cell Sci. 119: 4315-4321.

Kassabov, S.R., Zhang, B., Persinger, J., and Bartholomew, B. 2003. SWI/SNF unwraps, slides, and rewraps the nucleosome. Mol. Cell 11: 391-403.

Kaynak, B., von Heydebreck, A., Mebus, S., Seelow, D., Hennig, S., Vogel, J., Sperling, H.P., Pregla, R., Alexi-Meskishvili, V., Hetzer, R., et al. 2003. Genome-wide array analysis of normal and malformed human hearts. Circulation 107: 24672474.

Kel, A.E., Gossling, E., Reuter, I., Cheremushkin, E., Kel-Margoulis, O.V., and Wingender, E. 2003. MATCH: A tool for searching transcription factor binding sites in DNA se- 
quences. Nucleic Acids Res. 31: 3576-3579.

Kim, J., Daniel, J., Espejo, A., Lake, A., Krishna, M., Xia, L., Zhang, Y., and Bedford, M.T. 2006. Tudor, MBT and chromo domains gauge the degree of lysine methylation. EMBO Rep. 7: 397-403.

Kouzarides, T. 2007. Chromatin modifications and their function. Cell 128: 693-705.

Lan, F., Collins, R.E., De Cegli, R., Alpatov, R., Horton, J.R., Shi, X., Gozani, O., Cheng, X., and Shi, Y. 2007. Recognition of unmethylated histone $\mathrm{H} 3$ lysine 4 links $\mathrm{BHC} 80$ to LSD1mediated gene repression. Nature 448: 718-722.

Lessard, J., Wu, J.I., Ranish, J.A., Wan, M., Winslow, M.M., Staahl, B.T., Wu, H., Aebersold, R., Graef, I.A., and Crabtree, G.R. 2007. An essential switch in subunit composition of a chromatin remodeling complex during neural development. Neuron 55: 201-215.

Li, H., Fischle, W., Wang, W., Duncan, E.M., Liang, L., Murakami-Ishibe, S., Allis, C.D., and Patel, D.J. 2007. Structural basis for lower lysine methylation state-specific readout by MBT repeats of L3MBTL1 and an engineered PHD finger. Mol. Cell 28: 677-691.

Li, Z.Y., Yang, J., Gao, X., Lu, J.Y., Zhang, Y., Wang, K., Cheng, M.B., Wu, N.H., Wu, Z., and Shen, Y.F. 2007. Sequential recruitment of PCAF and BRG1 contributes to myogenin activation in 12-O-tetradecanoylphorbol-13-acetate-induced early differentiation of rhabdomyosarcoma-derived cells. $J$. Biol. Chem. 282: 18872-18878.

Lickert, H., Takeuchi, J.K., von Both, I., Walls, J.R., McAuliffe, F., Lee Adamson, S., Mark Henkelman, R., Wrana, J.L., Rossant, J., and Bruneau, B.G. 2004. Baf60c is essential for function of BAF chromatin remodelling complexes in heart development. Nature 432: 107-112.

Ma, L., Lu, M.F., Schwartz, R.J., and Martin, J.F. 2005. Bmp2 is essential for cardiac cushion epithelial-mesenchymal transition and myocardial patterning. Development 132: 56015611.

Mertsalov, I.B., Kulikova, D.A., Alimova-Kost, M.V., Ninkina, N.N., Korochkin, L.I., and Buchman, V.L. 2000. Structure and expression of two members of the $\mathrm{d} 4$ gene family in mouse. Mamm. Genome 11: 72-74.

Min, J., Allali-Hassani, A., Nady, N., Qi, C., Ouyang, H., Liu, Y., MacKenzie, F., Vedadi, M., and Arrowsmith, C.H. 2007. L3MBTL1 recognition of mono- and dimethylated histones. Nat. Struct. Mol. Biol. 14: 1229-1230.

Mohrmann, L. and Verrijzer, C.P. 2005. Composition and functional specificity of SWI2/SNF2 class chromatin remodeling complexes. Biochim. Biophys. Acta 1681: 59-73.

Mujtaba, S., Zeng, L., and Zhou, M.M. 2007. Structure and acetyl-lysine recognition of the bromodomain. Oncogene 26: 5521-5527.

Natalia, N.N., Ilja, B.M., Dina, A.K., Maria, V.A.-K., Olga, B.S., Leonid, I.K., Sergey, L.K., and Vladimir, L.B. 2001. Cerd4, third member of the d4 gene family: Expression and organization of genomic locus. Mamm. Genome V12: 862-866.

Naya, F.J., Black, B.L., Wu, H., Bassel-Duby, R., Richardson, J.A., Hill, J.A., and Olson, E.N. 2002. Mitochondrial deficiency and cardiac sudden death in mice lacking the MEF2A transcription factor. Nat. Med. 8: 1303-1309.

Palacios, D. and Puri, P.L. 2006. The epigenetic network regulating muscle development and regeneration. J. Cell. Physiol. 207: 1-11.

Peterson, C.L. and Workman, J.L. 2000. Promoter targeting and chromatin remodeling by the SWI/SNF complex. Curr. Opin. Genet. Dev. 10: 187-192.

Potthoff, M.J., Arnold, M.A., McAnally, J., Richardson, J.A., Bassel-Duby, R., and Olson, E.N. 2007. Regulation of skeletal muscle sarcomere integrity and postnatal muscle function by Mef2c. Mol. Cell. Biol. 27: 8143-8151.

Ramon-Maiques, S., Kuo, A.J., Carney, D., Matthews, A.G., Oettinger, M.A., Gozani, O., and Yang, W. 2007. The plant homeodomain finger of RAG2 recognizes histone $\mathrm{H} 3$ methylated at both lysine-4 and arginine-2. Proc. Natl. Acad. Sci. 104: 18993-18998.

Rauch, C. and Loughna, P.T. 2005. Static stretch promotes MEF2A nuclear translocation and expression of neonatal myosin heavy chain in $\mathrm{C} 2 \mathrm{C} 12$ myocytes in a calcineurinand p38-dependent manner. Am. J. Physiol. Cell Physiol. 288: C593-C605. doi: 10.1152/ajpcell.00346.2004.

Rottbauer, W., Baker, K., Wo, Z.G., Mohideen, M.A., Cantiello, H.F., and Fishman, M.C. 2001. Growth and function of the embryonic heart depend upon the cardiac-specific L-type calcium channel $\alpha 1$ subunit. Dev. Cell 1: 265-275.

Ruthenburg, A.J., Allis, C.D., and Wysocka, J. 2007. Methylation of lysine 4 on histone H3: Intricacy of writing and reading a single epigenetic mark. Mol. Cell 25: 15-30.

Schafer, D.A., Hug, C., and Cooper, J.A. 1995. Inhibition of CapZ during myofibrillogenesis alters assembly of actin filaments. J. Cell Biol. 128: 61-70.

Shi, X., Hong, T., Walter, K.L., Ewalt, M., Michishita, E., Hung, T., Carney, D., Pena, P., Lan, F., Kaadige, M.R., et al. 2006. ING2 PHD domain links histone H3 lysine 4 methylation to active gene repression. Nature 442: 96-99.

Shi, X., Kachirskaia, I., Walter, K.L., Kuo, J.H., Lake, A., Davrazou, F., Chan, S.M., Martin, D.G., Fingerman, I.M., Briggs, S.D., et al. 2007. Proteome-wide analysis in Saccharomyces cerevisiae identifies several PHD fingers as novel direct and selective binding modules of histone $\mathrm{H} 3$ methylated at either lysine 4 or lysine 36. J. Biol. Chem. 282: 2450-2455.

Sif, S. 2004. ATP-dependent nucleosome remodeling complexes: Enzymes tailored to deal with chromatin. I. Cell. Biochem. 91: 1087-1098.

Simone, C. 2006. SWI/SNF: The crossroads where extracellular signaling pathways meet chromatin. I. Cell. Physiol. 207: 309-314.

Simone, C., Forcales, S.V., Hill, D.A., Imbalzano, A.N., Latella, L., and Puri, P.L. 2004. p38 pathway targets SWI-SNF chromatin-remodeling complex to muscle-specific loci. Nat. Genet. 36: 738-743.

Sperling, S. 2007. Transcriptional regulation at a glance. BMC Bioinformatics 8: S2. doi: 10.1186/1471-2105-8-S6-S2.

Sussman, M.A., Baque, S., Uhm, C.S., Daniels, M.P., Price, R.L., Simpson, D., Terracio, L., and Kedes, L. 1998. Altered expression of tropomodulin in cardiomyocytes disrupts the sarcomeric structure of myofibrils. Circ. Res. 82: 94-105.

Takeuchi, J.K., Lickert, H., Bisgrove, B.W., Sun, X., Yamamoto, M., Chawengsaksophak, K., Hamada, H., Yost, H.J., Rossant, J., and Bruneau, B.G. 2007. Baf60c is a nuclear Notch signaling component required for the establishment of left-right asymmetry. Proc. Natl. Acad. Sci. 104: 846-851.

Toedling, J., Skylar, O., Krueger, T., Fischer, J.J., Sperling, S., and Huber, W. 2007. Ringo-An R/bioconductor package for analyzing ChIP-chip readouts. BMC Bioinformatics 8: 443. doi: 10.1186/1471-2105-8-221.

Vermeulen, M., Mulder, K.W., Denissov, S., Pijnappel, W.W., van Schaik, F.M., Varier, R.A., Baltissen, M.P., Stunnenberg, H.G., Mann, M., and Timmers, H.T. 2007. Selective anchoring of TFIID to nucleosomes by trimethylation of histone $\mathrm{H} 3$ lysine 4. Cell 131: 58-69.

Wang, Y.X., Qian, L.X., Yu, Z., Jiang, Q., Dong, Y.X., Liu, X.F., Yang, X.Y., Zhong, T.P., and Song, H.Y. 2005. Requirements of myocyte-specific enhancer factor $2 \mathrm{~A}$ in zebrafish cardiac contractility. FEBS Lett. 579: 4843-4850. 
Lange et al.

Wang, Y.X., Qian, L.X., Liu, D., Yao, L.L., Jiang, Q., Yu, Z., Gui, Y.H., Zhong, T.P., and Song, H.Y. 2007. Bone morphogenetic protein-2 acts upstream of myocyte-specific enhancer factor 2 a to control embryonic cardiac contractility. Cardiovasc. Res. 74: 290-303.

Westerfield, M. 1994. The zebrafish book. University of Oregon Press, Eugene, OR.

Wilkinson, D.G. and Nieto, M.A. 1993. Detection of messenger RNA by in situ hybridization to tissue sections and whole mounts. Methods Enzymol. 225: 361-373.

Wysocka, J., Swigut, T., Xiao, H., Milne, T.A., Kwon, S.Y., Landry, J., Kauer, M., Tackett, A.J., Chait, B.T., Badenhorst, P., et al. 2006. A PHD finger of NURF couples histone H3 lysine 4 trimethylation with chromatin remodelling. Nature 442: 86-90.

Yelon, D., Horne, S.A., and Stainier, D.Y.R. 1999. Restricted expression of cardiac myosin genes reveals regulated aspects of heart tube assembly in zebrafish. Dev. Biol. 214: 23-37. 


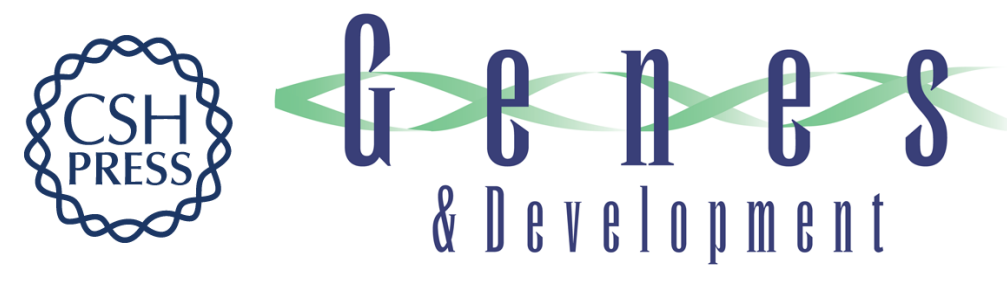

\section{Regulation of muscle development by DPF3, a novel histone acetylation and methylation reader of the BAF chromatin remodeling complex}

Martin Lange, Bogac Kaynak, Ulrike B. Forster, et al.

Genes Dev. 2008, 22:

Access the most recent version at doi:10.1101/gad.471408

Supplemental http://genesdev.cshlp.org/content/suppl/2008/08/14/22.17.2370.DC1

Material

References This article cites 63 articles, 19 of which can be accessed free at:

http://genesdev.cshlp.org/content/22/17/2370.full.html\#ref-list-1

License

Email Alerting Receive free email alerts when new articles cite this article - sign up in the box at the top

Service right corner of the article or click here.

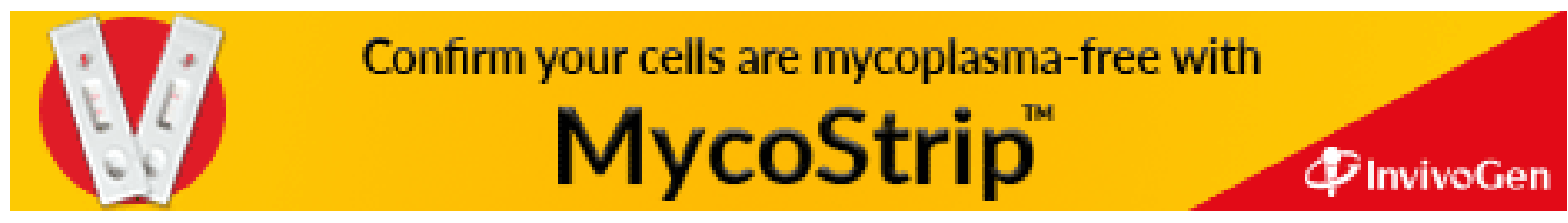

